\title{
Selective Serotonin Reuptake-Inhibitors for Symptom-Based Treatment of Borderline Personality Disorders in Older Adults: An International Delphi Study
}

\author{
Julie Schulkens ${ }^{1,2}$, Nina Bergs ${ }^{3}$, Theo Ingenhoven ${ }^{4}$, Erlene Rosowsky, ${ }^{5,6}$, Sebastiaan van Alphen ${ }^{1,7,8}$, \\ Sjacko Sobczak ${ }^{1,2}$ \\ ${ }^{1}$ Clinical Center of Excellence for Personality Disorders in Older Adults, Mondriaan Hospital, Heerlen, ${ }^{2}$ School for Mental Health and \\ Neuroscience (MHeNs), Department of Psychiatry and Neuropsychology, Maastricht University Medical Centre+, Maastricht, ${ }^{3}$ Faculty of Health \\ Medicine and Life Sciences, Maastricht University, Maastricht, ${ }^{4}$ Arkin Mental Healthcare, NPI Center for Personality Disorders, Amsterdam, \\ The Netherlands, ${ }^{5}$ Department of Clinical Psychology, William James College, Newton, MA, ${ }^{6}$ Department of Psychiatry, Harvard Medical School, \\ Boston, MA, USA, ${ }^{7}$ Department of Clinical and Lifespan Psychology, Vrije Universiteit Brussel, Brussels, Belgium, ${ }^{8}$ Department of Medical and \\ Clinical Psychology, Tilburg University, Tilburg, The Netherlands
}

\begin{abstract}
Objective: International guidelines on symptom-based treatment of borderline personality disorders (BPD) in older adults are lacking. The number of older adults ( $\geq 65$ years) with borderline personality disorder is rising. Effectiveness of Selective Serotonin Reuptake Inhibitors (SSRIs) on symptoms of BPD has only been investigated in younger adults and results are ambiguous. During life, serotonergic function changes, which can influence the indication and effectiveness of SSRIs in older adults with BPD. Aim of this study is to reach consensus on the suitability of SSRIs for the treatment of older adults with BPD.

Methods: A Delphi study was conducted among eighteen international experts. In three successive rounds, a total of 16 statements addressing the treatment with SSRI's in older adults with BPD were assessed. Consensus on specific statements was reached if at least two-third of these experts agreed.

Results: Consensus was reached on 11 statements related to the indication and effectiveness of SSRIs in the treatment of older adults with BPD.

Conclusion: The results of this study suggest a valuable role for SSRIs in the treatment of affective instability, and to a lesser extent impulsive behavior, in older adults with BPD. Sertraline or citalopram are suggested to be the first-choice medication but should be prescribed with some caution. Treatment recommendations have been suggested (presented in a flowchart), but still have to be investigated in clinical practice.
\end{abstract}

KEY WORDS: Delphi technique; Aged; Borderline personality disorder; Serotonin uptake inhibitors; Drug therapy.

\section{INTRODUCTION}

In recent years, interest in the treatment of borderline personality disorder (BPD) in older adults ( $\geq 65$ years) has steadily grown, partly due to ongoing ageing of the population. Globally, older adults are becoming a rela-

Received: June 16, 2020 / Revised: August 3, 2020

Accepted: August 5, 2020

Address for correspondence: Julie Schulkens

Clinical Center of Excellence for Personality Disorders in Older

Adults, Mondriaan Hospital, Kloosterkensweg 10, PO Box 4436,

6401 CX Heerlen, The Netherlands

E-mail: j.schulkens@mondriaan.eu

ORCID: https://orcid.org/0000-0002-0279-4137 tively larger share of society. Personality disorders (PDs) are highly prevalent in older adults; reported rates vary from. $10.6-14.5 \%$ in community dwelling older adults to $57.8 \%$ in nursing home-residents [1].

By all means, as people continue to age, the number of older adults with PDs will increase. While psychotherapy is the first-choice treatment for PDs, it can become less fitting to older adults, due to high prevalence of physical illness, cognitive impairment and disability, which can impair coping abilities $[2,3]$. In that case, pharmacotherapy can be helpful. Pharmacotherapy in older adults can be challenging; polypharmacy (defined as taking five or more drugs daily) [4] increases substantially with age and

(c) This is an Open-Access article distributed under the terms of the Creative Commons Attribution Non-Commercial License (http://creativecommons.org/licenses/by-nc/4.0) which permits unrestricted non-commercial use, distribution, and reproduction in any medium, provided the original work is properly cited. 
multimorbidity [5] and can cause drug-drug interactions [6], adverse drug events [7], falling [8] and cognitive impairment [9].

Studies on pharmacotherapy in patients with BPD mainly investigate younger adults, aged up to 50 years. The results are ambiguous [10]. A randomized controlled trial suggests SSRIs are more effective than antipsychotics in decreasing symptoms of depression, obsession and hypersensitivity in interpersonal relationships in adults with $\mathrm{BPD}$, and are effective in ameliorating symptoms of anxiety, somatization and aggression [11]. A review however, found no significant effects of SSRIs on any symptoms of BPD and concluded that SSRIs should only be used to treat psychiatric comorbidities [12].

The role of serotonin (5-HT) in BPD is not yet clear. Soloff et al. [13] found increased $5 \mathrm{HT}_{2 \mathrm{~A}}$ receptor binding in female BPD patients, compared to healthy controls. This could be explained by diminished serotonergic agonism causing postsynaptic up-regulation of $5 \mathrm{HT}_{2 \mathrm{~A}}$. Increased cortical $5 \mathrm{HT}_{2}$ binding has also been linked to higher levels of dysfunctional (more pessimistic) attitudes in depressed patients [14]. In older adults the role of 5-HT in BPD has not yet been investigated, but it is known that the availability of the $5-\mathrm{HT}$ precursor tryptophan decreases [15]. It is possible this could contribute to diminished serotonergic agonism and therefore affective instability, a common feature of BPD. However, this should be further investigated.

No studies so far have investigated the effect of SSRIs on affective instability in older adults with PDs, but several studies found SSRIs effective in treating late life depression $[16,17]$. In general SSRIs are relatively well tolerated by older adults [18]. Older adults seem to be somewhat more prone to some specific SSRI side-effects, such as hyponatremia [19] and risk of falling [20]. Citalopram and sertraline have been described as SSRIs with few interactions with other medications [21] and are in general well tolerated in older adults [22], which make them appropriate for pharmacotherapy in older adults.

This Delphi study is the first study on SSRI treatment in older adults with BPD. The main goal of this study is to reach consensus on the suitability of SSRIs for the treatment of older adults with BPD.

\section{METHODS}

A Delphi study includes a method that, in subsequent rounds, uses relevant statements to reach consensus among experts. It deals with a subject on which there is not much - or any - scientific knowledge yet [23]. Through this method, expert knowledge is generated and statements are tested in order to realize a design - based on an evaluation - for a practically applicable treatment protocol. The 16 statements (Table 1) in this Delphi study were compiled through a literature search and supplemented with the research team's clinical experience. Each statement was assessed individually (by means of a five-point Likert scale). Next, additional feedback was given by the experts. After each round, the results were analysed and assessed per statement. Consensus was reached when $\geq$ $66,6 \%$ of the participants agreed (strongly agree and agree) [24]. When no consensus was reached, the statement was adjusted and presented again in a next round. In these successive rounds, the experts were confronted with de-identified answers from other experts in former rounds. After each round, all experts received a brief summary of the results and were encouraged to give their opinion on the remaining and adjusted statements in order to try to reach any consensus on relevant topics.

\section{Recruitment of Experts}

The experts in this study were recommended by the Dutch-Flemish Personality \& Older Adults Expert Panel (EPO), a panel of international psychiatrists, specialists in geriatric medicine, psychologists and social psychiatric nurses holding important positions within geriatric psychiatry. The expert group consisted of intercontinental psychiatrists and geriatric physicians who needed to meet the following criteria: (1) they had demonstrable experience with treating and/or researching PDs; (2) they had at least 5 years of clinical, research, educational, diagnostic or treatment experience with geriatric patients with PDs; (3) they had clinical experience with the pharmacotherapy of older adults. This information was validated by the research team on questionnaires completed by these experts. In total, 27 potential international experts were identified. Of these 27 identified experts, 5 experts did not respond, and 3 experts declined the invitation to participate. Of the initial 19 experts who agreed to participate, one expert did not respond during the first round 
Table 1. Original statements

Statement 1. Pharmacotherapy is indicated as part of the treatment plan for elderly patients ( $\geq 65$ years) with a borderline personality disorder if it is expected that psychotherapy alone will not be sufficiently effective.

Statement 2 . The use of adjuvant pharmacotherapy in elderly patients ( $\geq 65$ years) with borderline personality disorder is indicated if it is expected that the nature, severity or persistence of the symptoms will compromise the process of the psychotherapeutic treatment.

Statement 3. The symptom clusters of borderline personality disorder in elderly patients ( $\geq 65$ years) can be divided into; cognitive-perceptual symptoms, affective dysregulation, and impulsive behavior.

Statement 4. In elderly patients ( $\geq 65$ years) with a borderline personality disorder, the use of adjuvant pharmacotherapy is indicated for the treatment of persistent or recurrent symptoms within these symptom clusters (cognitive-perceptual symptoms, affective dysregulation, and impulsive behavior).

Statement 5. For the treatment of cognitive-perceptual symptoms using a selective serotonin reuptake inhibitor (SSRI) is indicated in elderly patients ( $\geq 65$ years) with a borderline personality disorder.

Statement 6 . For the treatment of affective dysregulation using an SSRI is indicated in elderly patients ( $\geq 65$ years) with a borderline personality disorder.

Statement 7. For the treatment of impulsive behavior using an SSRI is indicated in elderly patients ( $\geq 65$ years) with a borderline personality disorder.

Statement 8. For the treatment of suicidal ideation and behaviors using an SSRI is indicated in elderly patients ( $\geq 65$ years) with a borderline personality disorder.

Statement 9 . The first choice for the pharmacotherapeutic treatment of elderly patients ( $\geq 65$ years) with a borderline personality disorder is citalopram or sertraline (SSRIs) because they are relatively safe for use with an elderly population.

Statement 10 . The optimal starting dose of citalopram for the treatment of elderly patients ( $\geq 65$ years) with a borderline personality disorder is 10 milligram once daily.

Statement 11 . The optimal starting dose of sertraline for the treatment of elderly patients ( $\geq 65$ years) with a borderline personality disorder is 50 milligram once daily.

Statement 12. A diagnosis of current hyponatremia is the absolute contraindication for treating elderly patients ( $\geq 65$ years) with a borderline personality disorder with an SSRI.

Statement 13. If treatment with an SSRI is successful (decrease of symptoms and the experience of distress) and is well tolerated in elderly patients ( $\geq 65$ years) with a borderline personality disorder, the treatment should be continued for life.

Statement 14. If after six weeks the treatment with the SSRI is not successful (symptoms and experience of distress not decreased) a trial with another SSRI is recommended.

Statement 15 . Gender affects the pharmacotherapeutic treatment in patients over 65 years with a borderline personality disorder as the symptom clusters are often expressed differently in men and women.

Statement 16. In men, SSRIs are particularly effective for impulsivity and aggression, while in women they are particularly effective for affective dysregulation.

Table 2. Countries of the experts

\begin{tabular}{lc}
\hline \multicolumn{1}{c}{ Country } & Participating experts \\
\hline The Netherlands & $4(22)$ \\
Belgium & $5(28)$ \\
United Kingdom & $3(17)$ \\
United States & $2(11)$ \\
Switzerland & $3(17)$ \\
Australia & $1(5)$ \\
Total & $18(100)$ \\
\hline
\end{tabular}

Values are presented as number (\%).

and was excluded from further study. Table 2 details the countries in which the participating experts were working during this study.

\section{Data Analysis}

Data were collected digitally by use of Qualtrics research software. After each round data were exported to an Excel spreadsheet. Using frequency, mean and stand-

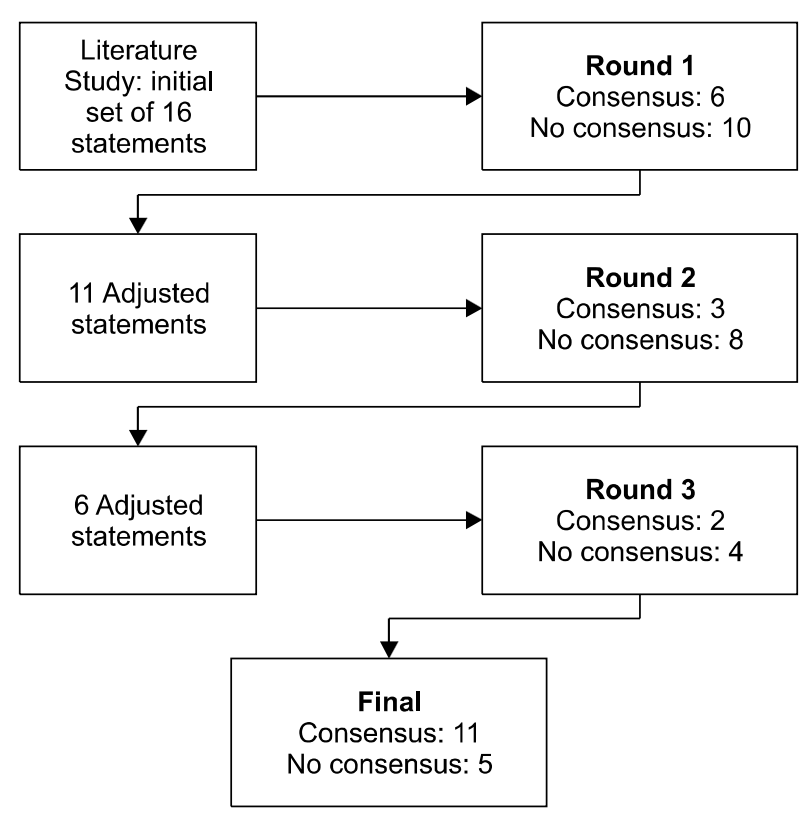

Fig. 1. Results per round. 
ard deviation formulas it was calculated per statement whether consensus had been reached.

\section{RESULTS}

This Delphi study consisted of three rounds, presented through e-mail and each round lasted 1.5 weeks. The response rate was $100 \%$. The experts were asked what literature they consulted to pharmacologically treat their current older patients with BPD. They indicated using different sources, such as the NICE Guideline [25], the pharmacotherapeutic algorithms in a Dutch Handbook of PDs [26] or literature such as 'Drug treatment for PDs' [27]. There were also experts who did not use any official guideline or algorithm. These experts prescribed their off-label medication based on their own knowledge, intuition and clinical experience. At the end of the study a consensus ( $\geq 66.6 \%$ agreement) was reached on 11 of the 16 initial statements (Fig. 1). Table 3 contains an extensive table with the results. After round one, consensus had been reached on 6 of the 16 initial statements. Amongst others, the experts agreed that pharmacotherapy is indicated as part of the treatment plan for older adults with BPD when it is anticipated that psychotherapy alone will not be sufficiently effective and when the nature, severity, or persistence of the symptoms will compromise the process of psychotherapeutic treatment. An SSRI is indicated for the treatment of affective instability, and their first choice SSRI is citalopram or sertraline. The experts also agreed that SSRIs are not indicated for the treatment of cognitive-perceptual symptoms. However, they can be used as a second option in treating impulsive behavior in older adults with a BPD. The remaining statements, on which no consensus was reached after three rounds, were statement $8,10,11,14$ and 16 (Table 3).

\section{DISCUSSION}

The main goal of this study was to reach consensus amongst international experts on the suitability of SSRIs for the treatment of older adults with BPD. The experts agreed on the statements that SSRIs are indicated for treatment of affective instability and as a second choice for impulsive behavior in older adults with BPD. Preferred SSRIs in older adults are citalopram and sertraline, but no consensus was reached on optimal dose. Based on the 11 statements for which consensus was reached, we composed a flowchart with treatment recommendations, which is shown in Figure 2.

Available placebo controlled RCTs show conflicting results when it comes to the effectiveness of SSRIs in treating affective instability in adults $[28,29]$. They could be effective for mood swings [28], anger [30], irritability [31], and anxiety [32]. According to the experts in this Delphi study, among older adults with BPD SSRIs are effective in treating affective instability. This is supported by the demonstrated effectiveness of SSRIs in de treatment of late life depression [16,17].

The experts also concluded that SSRIs are not preferred as first choice for treating impulsive behavior, but they can be used as a second choice (item 7). In adults, SSRIs have demonstrated to be effective in treating impulsive behavior and aggression, mostly in men with BPD $[28,30]$. The experts agreed on the statement that it is unclear whether gender has an effect on pharmacotherapy in older adults with BPD.

The experts reached consensus on citalopram and sertraline being first choice SSRIs for older adults with BPD. The fact that citalopram and sertraline are SSRIs with few interactions with other medications is a relevant factor in older adults, who are exposed to polypharmacy frequently [5].

However, at the end of this Delphi study some statements did not reach consensus, such as the statement on the dosage of sertraline and citalopram. Sertraline appears to be as safe as a placebo for the risk of dizziness, which makes it very preferable for older adults [33]. More caution might be required when using citalopram. The Food and Drug Administration (FDA) issued a safety communication which proclaimed that daily dosages of citalopram should no longer exceed $40 \mathrm{mg}$ because of the risk of prolonged QT interval [34]. Even though this was not supported by a cohort study in veterans [35] frequent monitoring of patients at risk of prolonged QT interval is a requirement.

There was also no consensus reached on the treatment of suicidality with an SSRI. The study by Stone et al. [36] showed SSRIs could have a protective effect for suicidal ideation but it has no effect on suicidal behavior.

This Delphi study generally encourages more research, for example to clarify dosing strategies in older adults with PDs; starting dose, optimal therapeutic dose, maximum 


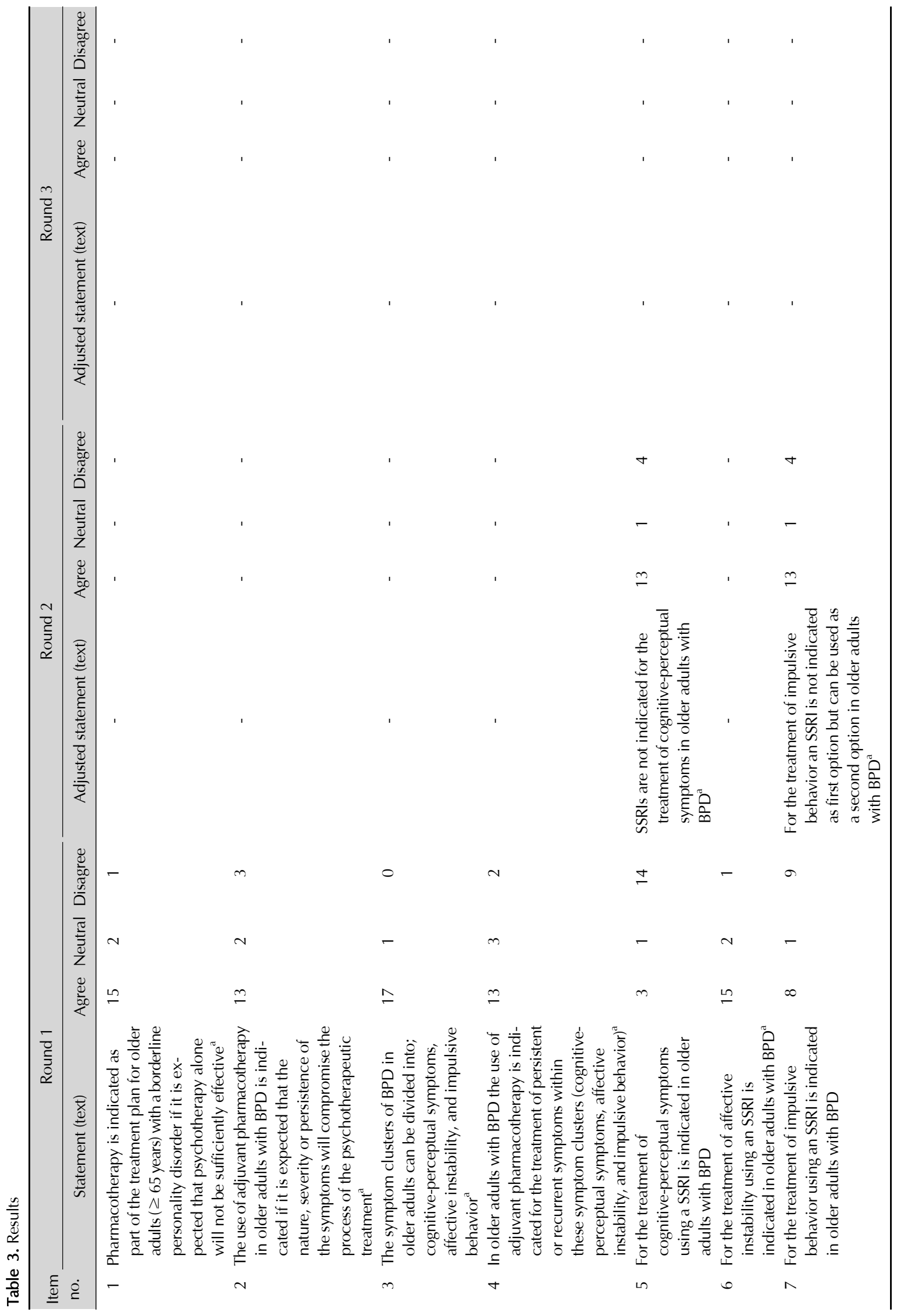




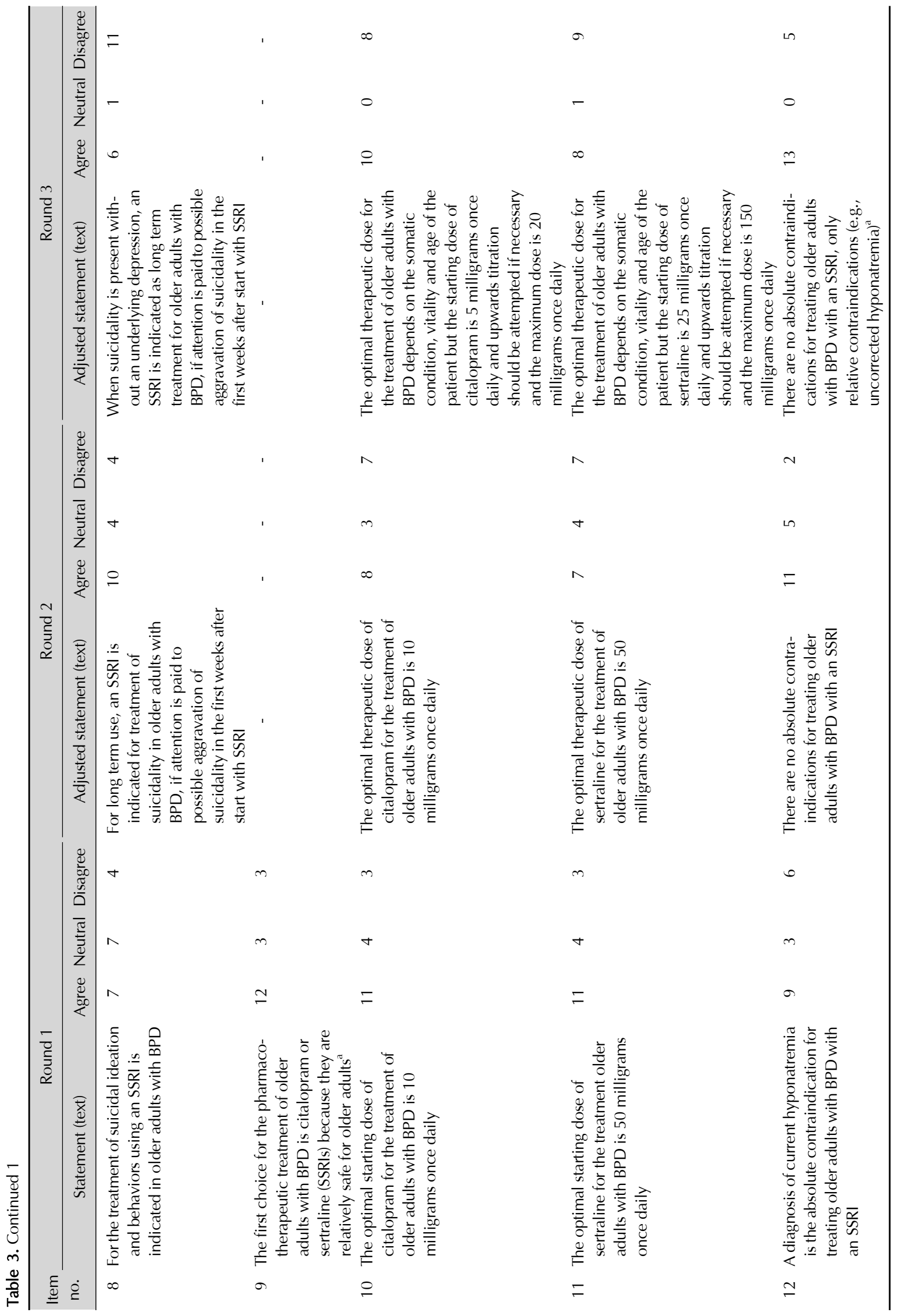




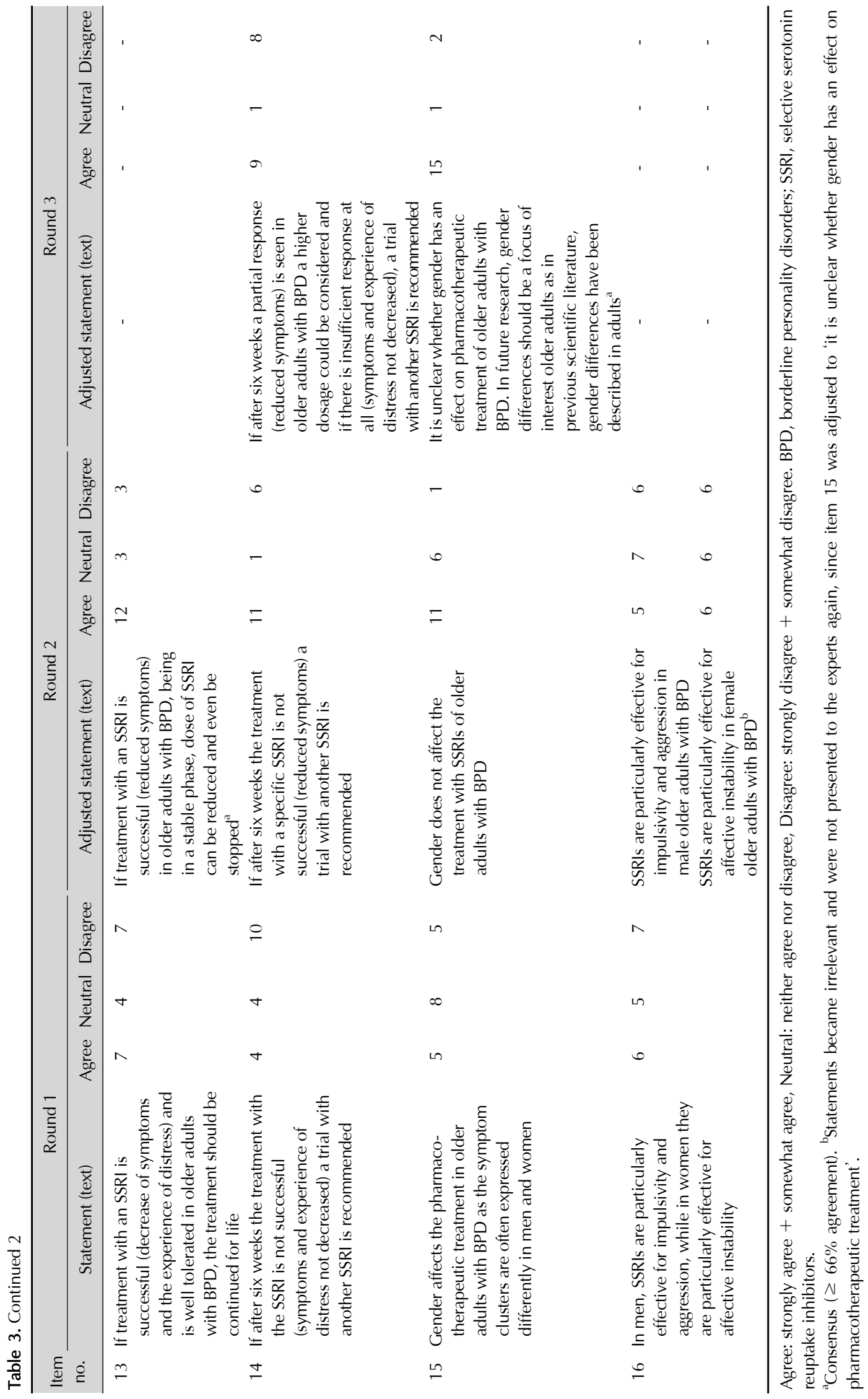




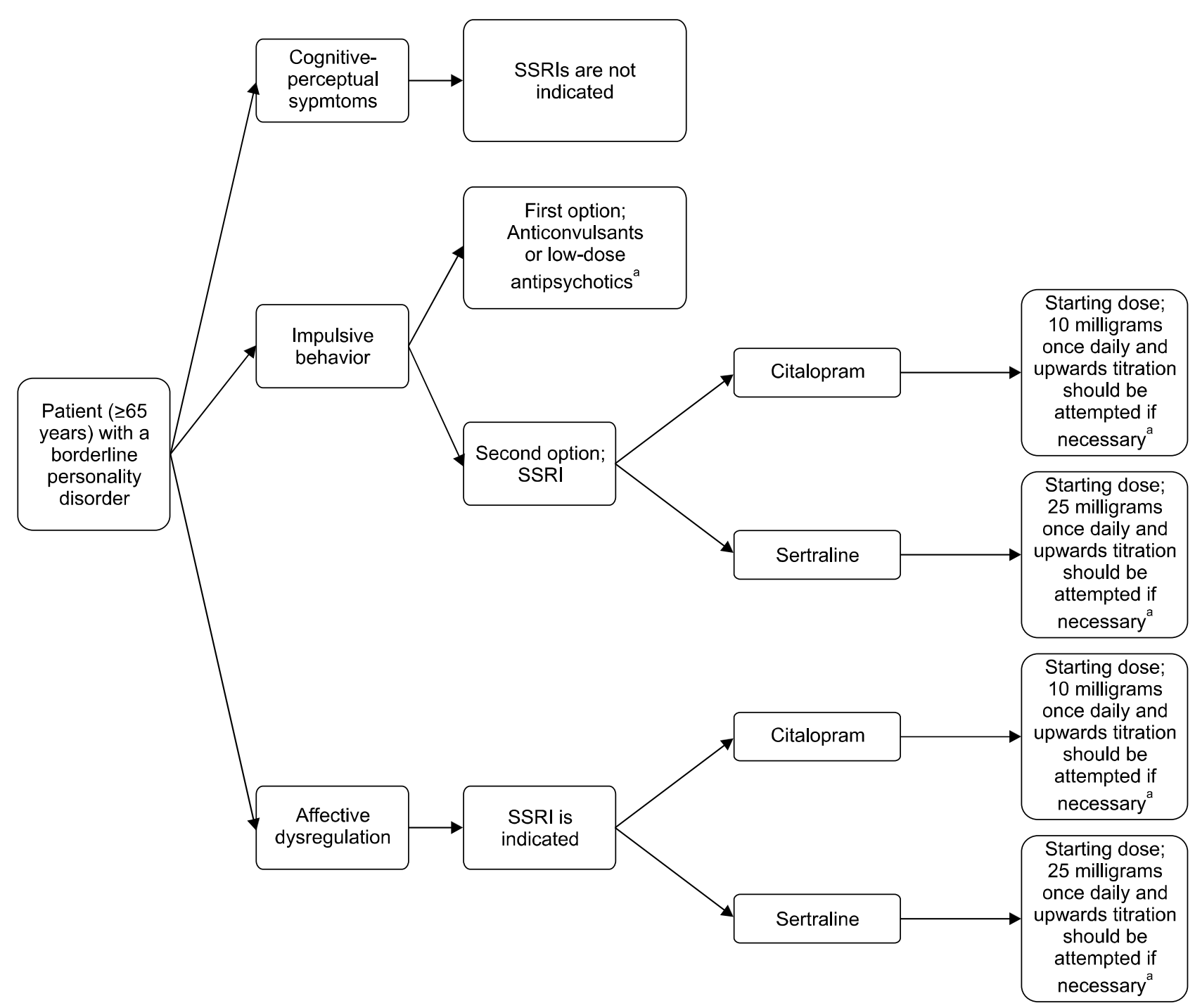

Fig. 2. Design for a treatment algorithm for older adults with BPD by means of an SSRI.

BPD, borderline personality disorders; SSRI, Selective Serotonin Reuptake Inhibitors.

If after six weeks a partial response (reduced symptoms) is seen, a higher dosage could be considered, and if there is insufficient response at all (symptoms and experience of distress not decreased), a trial with another SSRI is recommended or a change to another tricyclic antidepressant (nortriptyline) is recommended.a If the treatment with an SSRI is successful (reduced symptoms), being in a stable phase, the dose of SSRI can be reduced and even be stopped.

${ }^{\mathrm{a}}$ No consensus reached during the study.

dose, but also when to increase the dose of SSRIs. Most importantly, the first steps are made for treatment recommendations of older adults with BPD by use of an SSRI.

\section{Limitations}

In adherence to the findings, some limitations of the study must be considered. The Delphi method is a study design based on expert opinion and therefore has limited level of evidence. However, since no studies have yet been performed on the use of SSRIs in the treatment of
BPD in older adults, it is a valid approach to explore this new area of interest. In the first and second rounds there was much inclination towards the answer 'neither agree nor disagree'. Before the third round began the experts were asked to minimize the use of this answer. If this had been done from round 1 onwards, a consensus could have possibly been reached on statements that did not pass before. Although Delphi studies are most commonly performed with two or three rounds, it may have been useful to carry out a fourth round to achieve more 
consensus. Unfortunately, this was not possible in the reserved timeframe for this study. The subjects on which no consensus has been reached are leads for further research.

\section{Conclusions and Recommendations}

The results of this study suggest a valuable role for SSRIs in the treatment of affective symptoms, and to a lesser extent impulsive behavior, in older adults with BPD. Sertraline or citalopram are suggested to be the first choice medication, but should be prescribed with some caution. Treatment recommendations have been suggested (presented in a flowchart), but still have to be investigated in clinical practice. To test both effectiveness and tolerability of SSRIs in older adults with BPD we propose a single case experimental design, because it takes into account the heterogeneity of the population of older adults. This should eventually be investigated for other psychotropics too, such as antipsychotics and anticonvulsants. Evidence based pharmacotherapy is essential for an effective and safe treatment of the various symptoms of BPD in older adults.

\section{Conflicts of Interest}

No potential conflict of interest relevant to this article was reported.

\section{Author Contributions}

Designed the study: Sebastiaan van Alphen, Sjacko Sobczak. Assisted in designing the study: Julie Schulkens, Theo Ingenhoven, Erlene Rosowsky. Collected the data: Nina Bergs. Supervised data collection: Julie Schulkens, Sebastiaan van Alphen, Sjacko Sobczak. Assisted in writing the article: Theo Ingenhoven, Erlene Rosowsky, Sebastiaan van Alphen. Wrote the article: Julie Schulkens, Nina Bergs, Sjacko Sobczak.

\section{ORCID}

Julie Schulkens https://orcid.org/0000-0002-0279-4137

Nina Bergs

Theo Ingenhoven https://orcid.org/0000-0002-4050-1032

Erlene Rosowsky https://orcid.org/0000-0003-3926-4479 Sebastiaan van Alphen

https://orcid.org/0000-0001-7085-3349

Sjacko Sobczak https://orcid.org/0000-0001-6351-0479

\section{REFERENCES}

1. Penders KAP, Peeters IGP, Metsemakers JFM, van Alphen SPJ. Personality disorders in older adults: a review of epidemiology, assessment, and treatment. Curr Psychiatry Rep 2020;22:14.

2. Dombrovski AY, Mulsant BH, Houck PR, Mazumdar S, Lenze EJ, Andreescu C, et al. Residual symptoms and recurrence during maintenance treatment of late-life depression. J Affect Disord 2007; 103:77-82.

3. Williamson GM, Schulz R. Activity restriction mediates the association between pain and depressed affect: a study of younger and older adult cancer patients. Psychol Aging 1995; 10:369-378.

4. Masnoon N, Shakib S, Kalisch-Ellett L, Caughey GE. What is polypharmacy? A systematic review of definitions. BMC Geriatr 2017;17:230.

5. Slabaugh SL, Maio V, Templin M, Abouzaid S. Prevalence and risk of polypharmacy among the elderly in an outpatient setting: a retrospective cohort study in the Emilia-Romagna region, Italy. Drugs Aging 2010;27:1019-1028.

6. Doan J, Zakrzewski-Jakubiak H, Roy J, Turgeon J, Tannenbaum C. Prevalence and risk of potential cytochrome P450-mediated drug-drug interactions in older hospitalized patients with polypharmacy. Ann Pharmacother 2013;47:324-332.

7. Bourgeois FT, Shannon MW, Valim C, Mandl KD. Adverse drug events in the outpatient setting: an 11-year national analysis. Pharmacoepidemiol Drug Saf 2010;19:901-910.

8. Fried TR, O'Leary J, Towle V, Goldstein MK, Trentalange M, Martin DK. Health outcomes associated with polypharmacy in community-dwelling older adults: a systematic review. J Am Geriatr Soc 2014;62:2261-2272.

9. Jyrkkä J, Enlund H, Lavikainen P, Sulkava R, Hartikainen S. Association of polypharmacy with nutritional status, functional ability and cognitive capacity over a three-year period in an elderly population. Pharmacoepidemiol Drug Saf 2011; 20:514-522.

10. Ingenhoven T. Pharmacotherapy for borderline patients: business as usual or by default? J Clin Psychiatry 2015;76:e522e523.

11. Jariani $M$, Saaki $M$, Nazari $H$, Birjandi $M$. The effect of Olanzapine and Sertraline on personality disorder in patients with methadone maintenance therapy. Psychiatr Danub 2010;22:544-547.

12. Stoffers J, Völlm BA, Rücker G, Timmer A, Huband N, Lieb K. Pharmacological interventions for borderline personality disorder. Cochrane Database Syst Rev 2010;(6):CD005653.

13. Soloff PH, Chiappetta L, Mason NS, Becker C, Price JC. Effects of serotonin-2A receptor binding and gender on personality traits and suicidal behavior in borderline personality disorder. Psychiatry Res 2014;222:140-148.

14. Meyer JH, McMain S, Kennedy SH, Korman L, Brown GM, DaSilva JN, et al. Dysfunctional attitudes and 5-HT2 receptors during depression and self-harm. Am J Psychiatry 2003;160: 90-99. 
15. Bonaccorso S, Lin A, Song C, Verkerk R, Kenis G, Bosmans E, et al. Serotonin-immune interactions in elderly volunteers and in patients with Alzheimer's disease (DAT): lower plasma tryptophan availability to the brain in the elderly and increased serum interleukin-6 in DAT. Aging (Milano) 1998;10: 316-323.

16. Sheikh JI, Cassidy EL, Doraiswamy PM, Salomon RM, Hornig $\mathrm{M}$, Holland PJ, et al. Efficacy, safety, and tolerability of sertraline in patients with late-life depression and comorbid medical illness. J Am Geriatr Soc 2004;52:86-92.

17. Rapaport MH, Schneider LS, Dunner DL, Davies JT, Pitts CD. Efficacy of controlled-release paroxetine in the treatment of late-life depression. J Clin Psychiatry 2003;64:1065-1074.

18. Draper B, Berman K. Tolerability of selective serotonin reuptake inhibitors. Drugs \& Aging 2008;25:501-519.

19. Finfgeld DL. SSRI-related hyponatremia among aging adults. J Psychosoc Nurs Ment Health Serv 2003;41:12-16.

20. Laberge S, Crizzle AM. A literature review of psychotropic medications and alcohol as risk factors for falls in community dwelling older adults. Clin Drug Investig 2019;39:117-139.

21. Baldwin R, Wild R. Management of depression in later life. Advances Psychiatr Treat 2004;10:131-139.

22. Pinkowish MD, Block M, Gelenberg AJ, Malone DA. Rational use of the newer antidepressants. Patient Care 1997;31:49-62.

23. Linstone HA, Turoff M. The Delphi method: techniques and applications. Boston:Addison-Wesley Reading;1975.

24. Sharkey SB, Sharples AY. An approach to consensus building using the Delphi technique: developing a learning resource in mental health. Nurse Educ Today 2001;21:398-408.

25. National Collaborating Centre for Mental Health. Borderline personality disorder: treatment and management. London: British Psychological Society and the Royal College of Psychiatrists;2009.

26. Eurelings-Bontekoe EHM, Verheul R, Snellen WM. Handboek persoonlijkheidspathologie: voor opleiding, onderzoek en klinische praktijk. Berlin:Springer;2017.

27. Tyrer P, Bateman AW. Drug treatment for personality disorders. Advances Psychiatr Treat 2004;10:389-398.
28. Rinne T, van den Brink W, Wouters L, van Dyck R. SSRI treatment of borderline personality disorder: a randomized, placebo-controlled clinical trial for female patients with borderline personality disorder. Am J Psychiatry 2002;159:2048-2054.

29. Simpson EB, Yen S, Costello E, Rosen K, Begin A, Pistorello J, et al. Combined dialectical behavior therapy and fluoxetine in the treatment of borderline personality disorder. J Clin Psychiatry 2004;65:379-385.

30. Salzman C, Wolfson AN, Schatzberg A, Looper J, Henke R, Albanese $\mathrm{M}$, et al. Effect of fluoxetine on anger in symptomatic volunteers with borderline personality disorder. J Clin Psychopharmacol 1995; 15:23-29.

31. Coccaro EF, Kavoussi RJ. Fluoxetine and impulsive aggressive behavior in personality-disordered subjects. Arch Gen Psychiatry 1997;54:1081-1088.

32. Ingenhoven T, Lafay $\mathrm{P}$, Rinne $\mathrm{T}$, Passchier J, Duivenvoorden $\mathrm{H}$. Effectiveness of pharmacotherapy for severe personality disorders: meta-analyses of randomized controlled trials. York:Centre for Reviews and Dissemination (UK);1995.

33. Thorlund K, Druyts E, Wu P, Balijepalli C, Keohane D, Mills E. Comparative efficacy and safety of selective serotonin reuptake inhibitors and serotonin-norepinephrine reuptake inhibitors in older adults: a network meta-analysis. J Am Geriatr Soc 2015;63:1002-1009.

34. US Food \& Drug Administration. FDA Drug safety communication: revised recommendations for Celexa (citalopram hydrobromide) related to a potential risk of abnormal heart rhythms with high doses. Silver Spring:US Food \& Drug Administration;2012.

35. Zivin K, Pfeiffer PN, Bohnert AS, Ganoczy D, Blow FC, Nallamothu BK, et al. Evaluation of the FDA warning against prescribing citalopram at doses exceeding $40 \mathrm{mg}$. Am J Psychiatry 2013;170:642-650.

36. Stone $\mathrm{M}$, Laughren $\mathrm{T}$, Jones $\mathrm{ML}$, Levenson $\mathrm{M}$, Holland PC, Hughes $\mathrm{A}$, et al. Risk of suicidality in clinical trials of antidepressants in adults: analysis of proprietary data submitted to US Food and Drug Administration. BMJ 2009;339:62880. 